\title{
Influence of the conditions of selective laser melting on evaporation
}

\author{
Roman S. Khmyrov ${ }^{1, *}$, Cyrill E. Protasov ${ }^{1}$, and Andrey V. Gusarov ${ }^{1}$ \\ ${ }^{1}$ Moscow State University of Technology “STANKIN", 127055, Vadkovskii per. 1, Moscow, Russia
}

\begin{abstract}
The paper presents the results of optical diagnostics of evaporation and displacement of powder fractions during the formation of a single track in the process of selective laser melting. The velocity of the powder fractions is estimated. It was defined, that an increase in the scanning speed leads to an decrease in the particle coming out rate from the molten pool and the rate at which they are attracted. The results allow evaluating the kinetics of the mass-transfer process during selective laser melting. It was clearly shown the material quality properties after the selective laser melting are strongly influenced by the formed thermal field in the laser-irradiated zone.
\end{abstract}

\section{Introduction}

Laser material processing becomes already quite common in the modern industry, for example such processes as laser cutting, welding, drilling, marking. Technologies of additive manufacturing (growing) of parts from metal powders are also becoming more popular and characterizing by fundamentally different manufacturing strategy: layer by layer.

It should be mentioned, that the lack of theoretical apparatus describing the process technology is the fundamental problem in the field of laser treatment. Such fundamental problems which are necessary to solve are: powder consolidation kinetics and its dependence of process parameters, intensive powder release (slopping) from powder consolidation zone: its causes, methods of influence on it; physical mechanisms leading to geometry instability of fused bead, instability at overcharged and undercharged scanning speeds of the laser beam relative to the optimal values, mechanisms of integration of individual track in a single one. etc. Thereby, there are some difficulties in understanding the phenomena occurring during laser irradiation of the material.

The basis for active control systems developing is the results of the optical diagnostics of selective laser melting (SLM) process $[1,2]$. The previous works showed actual results: the real (not brightness) temperature estimating method was found, the process parameters influence on the track formation was evaluated [3], and the external conditions influence on the process was determined. The laser-irradiated area visualization and numerical simulation provides considering all physical factors and phenomena during the laser processing $[4,5]$. The present work is devoted to the combination of accumulated

\footnotetext{
${ }^{*}$ Corresponding author: r.khmyrov@stankin.ru
} 
knowledge and new scientific research in the field of control of track formation and process kinetics.

\section{Materials and methods}

Optical diagnostics was carried out on an experimental laboratory-scale selective laser melting machine developed in the laboratory of innovative additive technologies of MSTU "STANKIN" according to the scheme on the Fig. 1. The energy source was the fiber laser LK-200V (IPG-Photonics, Russia) with a maximum power is $200 \mathrm{~W}$, the radiation wavelength is $1070 \mathrm{~nm}$. The focal beam diameter is $100 \mu \mathrm{m}$, and the spot transverse energy distribution is similar to the Gaussian.

As a measuring device, a high-speed (HS) camera of the visible range Photron SA5 (USA) was used with a frequency of $10 \mathrm{kHz}$ (frames / s) (exposure $\approx 0.1 \mathrm{~ms}$ ) and a spatial resolution of $0.1 \mathrm{px} / \mu \mathrm{m}$, as well as infrared (IR) FLIR Phoenix RDAC camera (Flir, USA) (working wavelength range is $1100-1700 \mathrm{~nm}$ ) with a frequency of $340 \mathrm{~Hz}$ (exposure time: 3 $\mathrm{ms}$ ) and a resolution of 0.87 pixels / micron. The survey frequency and resolution was limited by the capacity of the data bus. To exclude the laser radiation exposure of the HScamera CCD-matrix, a notch-filter cuts off the wavelength of $1064 \pm 20 \mathrm{~nm}$ was used. Optical instruments were installed in the working chamber at an angle of at least $45^{\circ}$ to the surface of the substrate.

The molybdenum powder Mo 99,5\% (Amdry 313X, Oerlicon Metco) with powder fraction of 30-75 $\mu \mathrm{m}$ were used as the initial powders. As a substrate, the 321 steel plates of $4 \mathrm{~mm}$ were used in the work. The powder were produced by gas atomization and presented almost the spherical shape and homogeneous fraction distribution. The powder were sieved with 0.050 mesh (mesh cell is $50 \mu \mathrm{m}$ ) and dried in the vacuum oven at $120{ }^{\circ} \mathrm{C}$ for 2 hours. The substrate was sandblasted and the surface was cleaned with alcohol.

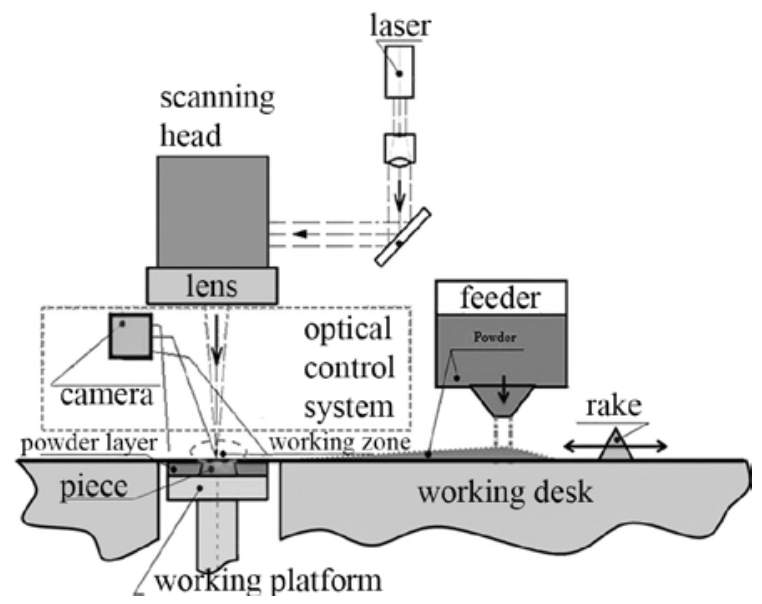

Fig. 1. Optical diagnostics equipment scheme coupled with SLM unit.

SLM optical diagnostics experiments were carried out with molybdenum powder with the following process parameters: powder leveling thickness was $100 \mu \mathrm{m}$, the laser power of 100 and $170 \mathrm{~W}$, the beam scanning speed of 75 and $100 \mathrm{~mm} / \mathrm{s}$. The received video data were collected, saved and analyzed. The particle velocity was calculated by the method described in the work [2]. 


\section{Results and discussion}

The results of the study are the rates of particle coming out from the molten pool and rates at which they are attracted The high-resolution images of the laser interaction zone at the scanning speeds 75 and $100 \mathrm{~mm} / \mathrm{s}$ shows in fig. 1. The laser beam moves perpendicular to the substrate from right to left. The central bright spot in each frame is identified as the melt pool. The melted track is visible to the right of the melt pool.

a

b
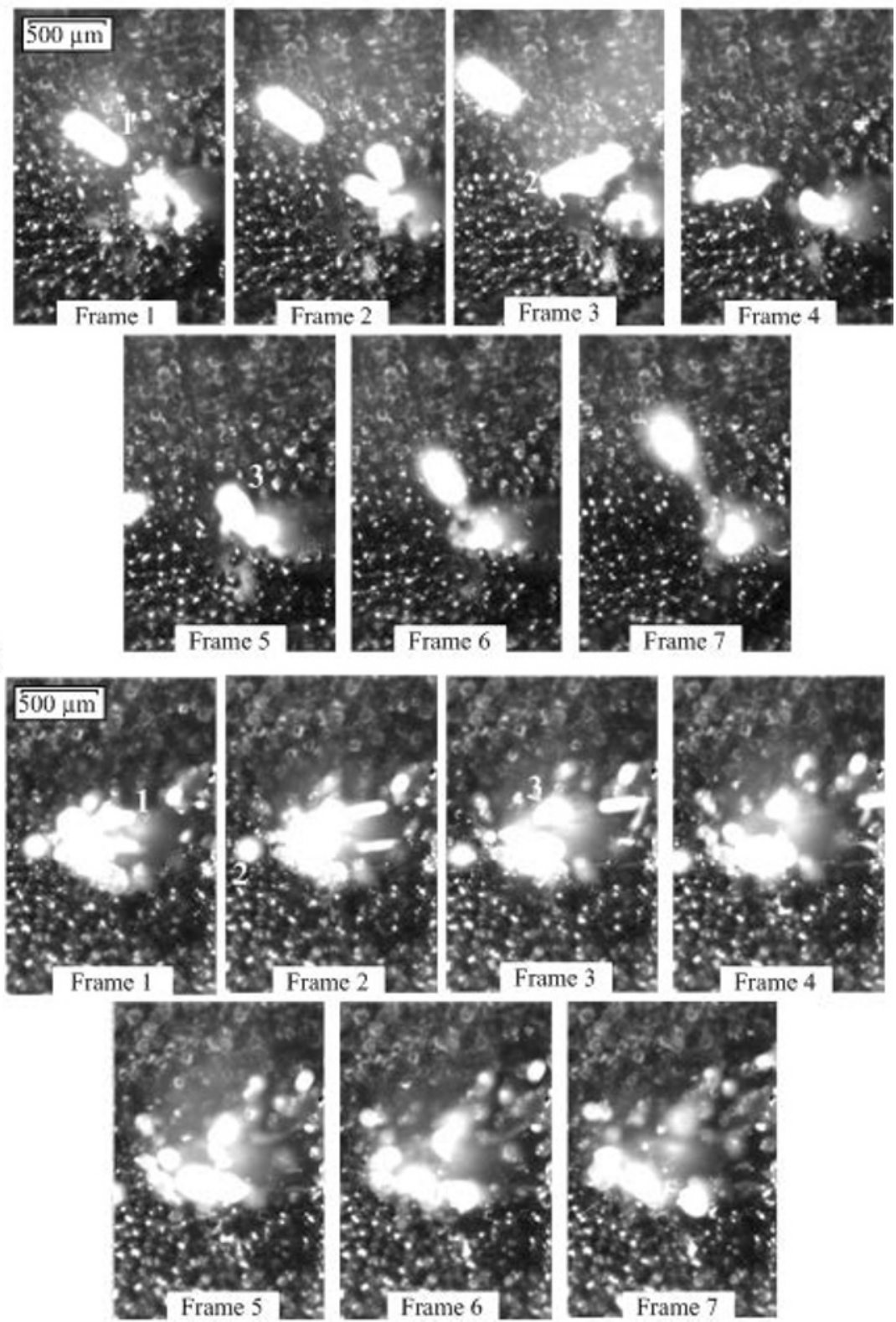

Fig. 2. Seven consecutive frames (numbered) of the laser interaction zone at SLM for two variants of scanning speeds: (a) is $75 \mathrm{~mm} / \mathrm{s}$, (b) $100 \mathrm{~mm}$ 
Consider the upper range of consecutive frames for the scanning speed is $75 \mathrm{~mm} / \mathrm{s}$ (Figure 2). In frame 1, the first particle emits from the melt pool. Its size is about $200 \mu \mathrm{m}$. The motion of this particle can be traced on frames 1,2 and 3. The trajectory of its motion is inclined at an angle of about $45^{\circ}$ to the scanning direction. However, its images are strongly elongated because it passes a considerable distance during the exposition of 100 $\mu \mathrm{s}$. The length of the particle's traces makes it possible to obtain the velocity of the particle and on frames 1,2 and 3 are $2.6 \mathrm{~m} / \mathrm{s}, 2.29 \mathrm{~m} / \mathrm{s}$ and $2.04 \mathrm{~m} / \mathrm{s}$, respectively. On frame 2, it can be seen that 2 particles of $200 \mu \mathrm{m}$ and $150 \mu \mathrm{m}$ sizes are emitted from the melt pool at angles of $60^{\circ}$ and $30^{\circ}$ degrees with velocities of about $1.1 \mathrm{~m} / \mathrm{s}$. On frame 3 , it can be seen that these two particles join together, sharply change direction and then fly along with the same speed. A third particle with a size of about $200 \mu \mathrm{m}$ appears in frame 5 from the melt pool. The speed determined from frames 5,6 and 7 are $1.52 \mathrm{~m} / \mathrm{s}, 1.47 \mathrm{~m} / \mathrm{s}$ and $1.35 \mathrm{~m} / \mathrm{s}$, respectively.

The figure 2 in the bottom range shows consecutive frames of the laser interaction zone at the scanning speed $100 \mathrm{~mm} / \mathrm{s}$. At this speed, there are a large flight of particles that fly in different directions and trajectory of their movement is very difficult to trace. The melt pool is practically not formed, but the cooled drops of metal remain. In frame 1 , it can be seen that a particle of about $100 \mu \mathrm{m}$ in size is emitted from the laser interaction zone. It does not move in the direction of the beam, but in the opposite direction along a very inclined trajectory with a speed of about $1.94 \mathrm{~m} / \mathrm{s}$ (frame 1), $1.76 \mathrm{~m} / \mathrm{s}$ (frame 2), $1.38 \mathrm{~m} / \mathrm{s}$ (frame 3 ). On the frame, 1 particle 2 with a size of $150 \mu \mathrm{m}$ slowly moves at a velocity of about 0.7 $\mathrm{m} / \mathrm{s}$ practically parallel to the direction of the beam. A particle 3 appears in frame 3 , which also moves in the opposite direction, but at a large angle (approximately $70^{\circ}$ ) than particle 1. The velocity of this particle, determined from frames $3,4,5$ and 6 , is $0.42 \mathrm{~m} / \mathrm{s}, 0.39 \mathrm{~m} / \mathrm{s}$, $0.36 \mathrm{~m} / \mathrm{s}$ and $0.29 \mathrm{~m} / \mathrm{s}$, respectively.

Figure 3 shows the time of flight $t$ of the droplet versus the distance $\mathrm{x}$ from the droplet center to the melt surface for the three droplets. The points are determined from the frames shown in Fig. 2. The uncertainty of the position of the droplet in Fig. 3 is about a few pixels. The pixel is $5 \mu \mathrm{m}$. The droplet velocities shown in Fig. 2 are obtained by two methods. The first is the numerical differentiation of the trajectory $t(x)$. In the second one, the length of the droplet trace in a frame is divided by the exposition time as explained above. The scatter of the two types of data indicates the uncertainty. Finally, for a scanning speed of $75 \mathrm{~mm} / \mathrm{s}$ the measured velocity of drops 1,2 and 3 is $2.3 \pm 0.2 \mathrm{~m} / \mathrm{s}, 1.1 \pm 0.1 \mathrm{~m} / \mathrm{s}$ and $1.4 \pm 0.1 \mathrm{~m} / \mathrm{s}$, respectively. For a scanning speed of $100 \mathrm{~mm} / \mathrm{s}$, the measured velocity of drops 1,2 and 3 is $1.9 \pm 0.2 \mathrm{~m} / \mathrm{s}, 0.7 \pm 0.2 \mathrm{~m} / \mathrm{s}$ and $0.4 \pm 0.1 \mathrm{~m} / \mathrm{s}$, respectively.

The high-speed visualization of the laser interaction zone reveals not only the ejected droplets but also a collective motion of the powder particles on the substrate toward the melt pool. The velocity of the droplet in the melt pool reaches about $0.25 \mathrm{~m} / \mathrm{s}$ at a scanning speed of $75 \mathrm{~mm} / \mathrm{s}$. At a scanning speed of $100 \mathrm{~m} / \mathrm{s}$, the powders on the substrate are practically not moved in the direction of the melt pool. Also, a feature of the involvement of particles in a molten pool along a parabolic trajectory was noted. Figure 4 shows the frames in which the particle attracted to the molten pool is casting a shadow onto the surface.

A comparison of the results obtained and the results of [3] allow us to say that with an increase in the scanning speed, the rate of ejected and the involvement of the powder fractions in the molten pool decreases. The number of such particles increases. It is noted that as the scanning speed increases, the trajectories of particle emission become multidirectional. 

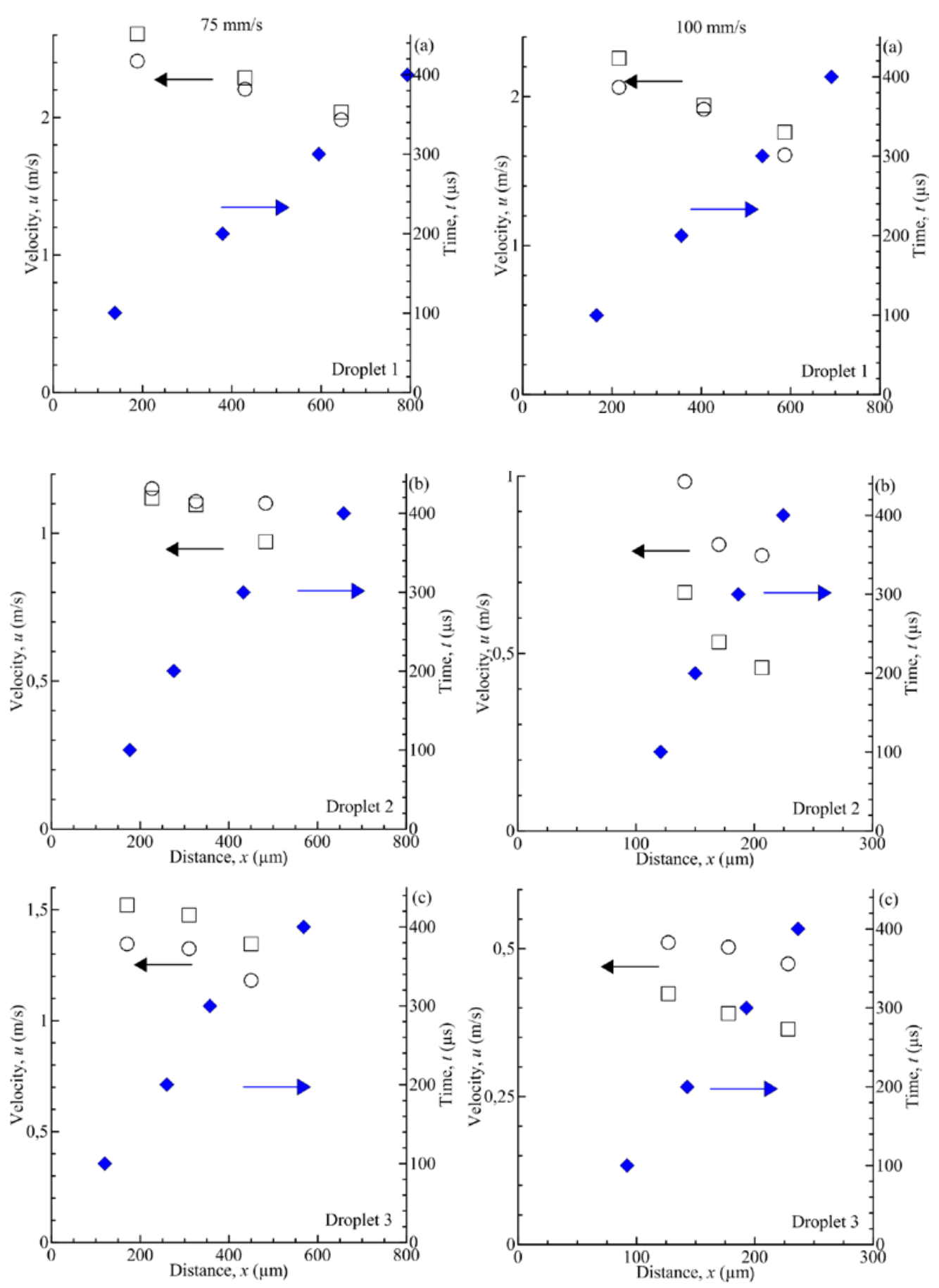

Fig. 3. The trajectories of drops 1 (a), 2 (b) and 3 (c) for two scanning speeds $t$ (x) (closed symbols), and their velocities $\mathrm{u}(\mathrm{x})$ (open symbols) 

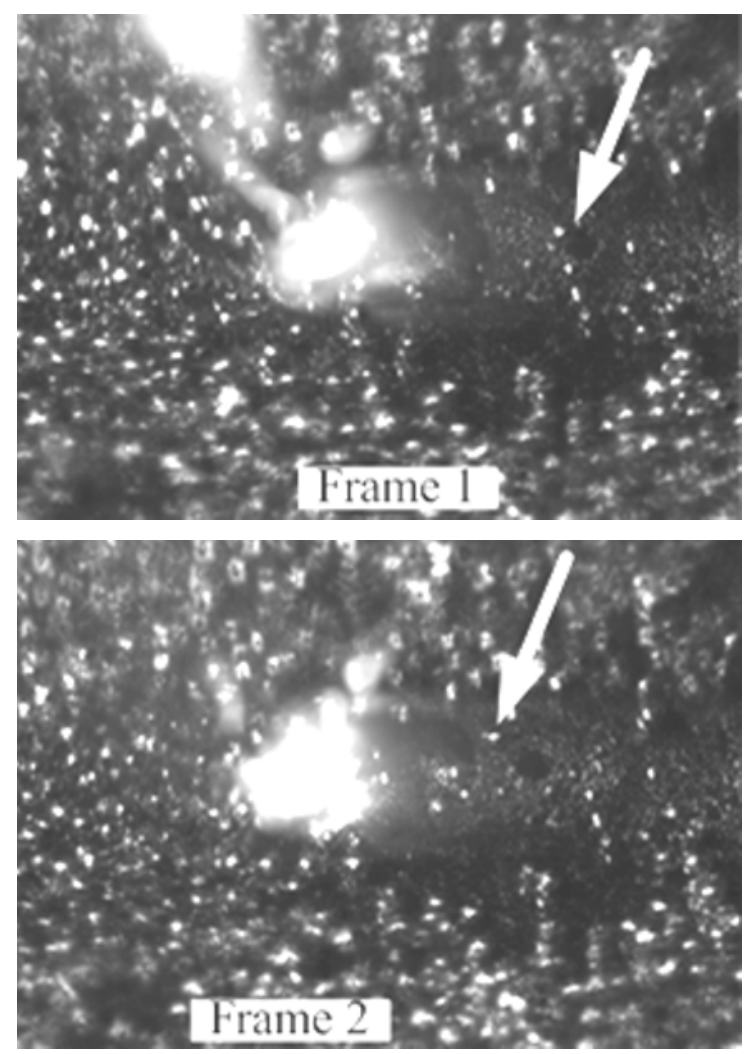

Fig. 4. Trajectory of the particle involved in the pool melt at a scanning speed of $75 \mathrm{~mm} / \mathrm{s}$

\section{Conclusion}

The use of diagnostic tools as the HS-camera and the IR-camera allows studying the fast processes occurring in the laser exposure zone, diagnosing the reasons of the particles motion leading to the formation of non-uniform distribution of the melted material volume.

The results of the study showed the kinetics of particles consolidation into a monolithic track. In the course of the study, the velocities of the particles involved in the melt pool and particles ejected from the treatment zone were calculated. The dependence of the scan rate on the particle coming out rate from the molten pool and the rate at which they are attracted is determined.

The achieved results will make it possible to reduce the complexity of the technological parameters optimization of selective laser melting, will make a fundamental knowledge of the phenomena occurring in selective laser melting.

This work has been financed by the sources of Russian Science Foundation (grant agreement №1519-00254). 


\section{References}

1. Gusarov, A. V., Okun'kova, A. A., Peretyagin, P. Y., Zhirnov, I. V., \& Podrabinnik, P. A. Means of Optical Diagnostics of Selective Laser Melting with Non-Gaussian Beams. Measurement Techniques, 58,8 (2015)

2. Zhirnov, I., D. V. Kotoban, \& A. V. Gusarov. "Evaporation-induced gas-phase flows at selective laser melting." Applied Physics, A124.2 (2018)

3. Khmyrov R.S., Safronov V.A.,. Gusarov A.V., Obtaining crack-free WC-Co alloys by selective laser melting, Phys.Procedia 83, 874-881 (2016)

4. Khmyrov, R. S., Protasov, C. E., Grigoriev, S. N., \& Gusarov, A. V. Crack-free selective laser melting of silica glass: single beads and monolayers on the substrate of the same material. The International Journal of Advanced Manufacturing Technology, 85 (2016)

5. Kovaleva, I. O., Grigoriev, S. N., \& Gusarov, A. V. Non-disturbing boundary conditions for modeling of laser material processing. Physics Procedia, 56, 421-428. (2014) 